\title{
Dificultades en la elección de una ecuación de referencia para la interpretación de los resultados de capacidad de difusión de monóxido de carbono
}

\author{
CATALINA BRICEÑO V.*, JORGE DREYSE D.*, LAURA MENDOZA I.**, \\ ORLANDO DÍAZ P.*, GESMA MERCADO M.* y GISELLA BORZONE T.*
}

\begin{abstract}
Difficulties in the selection of a reference equation for the interpretation of the results of DLCO
\end{abstract}

Introduction: In the absence of national reference equations, the ATS recommends comparing the results of the diffusion capacity of healthy subjects in a representative sample of the population with international equations and choosing among them, the one that provides the lowest sum of the residues. Objective: To compare reference equations available in the literature and identify which best meets the selection criteria. Methods: We reviewed 10 reference equations, for each one we calculated the sum of the residues for a sample of 71 healthy subjects and described the characteristics that affect the variability of each one. Results: Since 1985 we have used the single breath method. Only Thompson et al prediction equations 2008 were obtained with instantaneous reading of CO. Equations with the lowest sum of residues (Miller, Roca and Cotes) include smokers and former smokers. Conclusions: We need an equation in non-smokers with methodology that ensures low variability.

Key words: DLCO, lung function, reference equation, reference values.

\section{Resumen}

Introducción: En ausencia de ecuaciones de referencia nacionales, la ATS recomienda comparar los resultados de capacidad de difusión de monóxido de carbono de sujetos sanos en una muestra representativa de la población, con ecuaciones internacionales y escoger entre estas, aquella que presente la menor suma de los residuos. Objetivo: Comparar las ecuaciones de referencia disponibles en la literatura e identificar cuál cumple mejor los criterios de selección. Método: Revisamos 10 ecuaciones de referencia; calculamos la suma de los residuos de cada una de ellas para una muestra de 71 sujetos sanos y describimos las características que inciden en la variabilidad de cada una. Resultados: Desde 1985 se ha utilizado el método de respiración única. Sólo las ecuaciones de Thompson y cols. 2008 fueron obtenidas con lectura instantánea. Las ecuaciones que presentan menor suma de residuos (Miller, Roca y Cotes) incluyen fumadores y ex fumadores. Conclusiones: Es necesaria una ecuación nacional en sujetos sanos no fumadores, con metodología que asegure baja variabilidad.

Palabras clave: DLCO, función pulmonar, ecuación de referencia, valores teóricos.

\section{Introducción}

La medición de la capacidad de difusión de monóxido de carbono (DLCO) es un examen de uso común en el estudio de diversas enfermedades pulmonares y es una de las pocas pruebas que tiene como objetivo evaluar la transferencia de gases en el pulmón ${ }^{1}$.

Una disminución de la DLCO puede ser la manifestación de un gran número de enfermedades pulmonares (restrictivas, obstructivas, vasculares) y su realización sirve tanto para el diagnóstico, como para el seguimiento y la evaluación de la respuesta a diversos tratamientos ${ }^{2,3}$.

\footnotetext{
* Pontificia Universidad Católica de Chile.

** Hospital José Joaquín Aguirre, Universidad de Chile.
} 
Su medición se realiza con un paciente sentado que exhala hasta volumen residual y luego inhala una mezcla de monóxido de carbono $(0,3 \%)$, aire y gas trazador (habitualmente 1 a $5 \%$ de helio, aunque también se puede utilizar metano al 0,3\% o neón al $0,5 \%$ ), seguido de una apnea de 10 segundos y una exhalación rápida. La porción alveolar del gas espirado es recolectada para el análisis de la concentración tanto del $\mathrm{CO}$ como del gas trazador, existiendo 2 técnicas para dicho análisis. Inicialmente se utilizó el análisis del gas recolectado después de eliminar un volumen de espacio muerto estimado, método con una alta variabilidad, superior a la de la capacidad vital forzada ${ }^{4}$. Posteriormente, los avances tecnológicos permitieron el desarrollo de equipos con lectura instantánea de monóxido de carbono, que aseguran una muestra alveolar verdadera y gracias a ello, una medición de DLCO con una menor variabilidad $^{5}$. En la actualidad ambas técnicas de análisis se encuentran en equipos en uso en nuestro país.

Distintas sociedades científicas han contribuido a estandarizar el procedimiento de medición de la DLCO, a través del desarrollo de guías, con lo que se ha podido reducir la gran variabilidad entre un laboratorio y otro (hasta $50 \%$ ), permitiendo la comparación de resultados ${ }^{6,7}$.

En relación con la interpretación de los resultados, no existe una ecuación de referencia única que sea recomendada por la ATS (American Thoracic Society)/ERS (European Respiratory Society). En ausencia de valores de referencia que representen la población en estudio, el consenso ATS/ERS recomienda comparar los resultados de la capacidad de difusión de $\mathrm{CO}$ de sujetos sanos en una muestra representativa de la población con ecuaciones internacionales y escoger entre estas, aquella que presente la menor suma de los residuos $^{8}$. Sin embargo, algunas de las ecuaciones disponibles no sólo difieren entre sí, sino que han sido obtenidas con metodología distinta a la metodología en uso para la medición de la DLCO.

Nuestro objetivo fue comparar las ecuaciones de referencia disponibles en la literatura desde la aparición de las normas de estandarización de la ATS, analizando sus diferencias e identificando aquellas que cumplen mejor con los criterios de selección, utilizando una muestra de sujetos sanos de distinta talla, edad y género.

\section{Materiales y Método}

\section{Ecuaciones}

Se realizó una búsqueda bibliográfica en la base PubMed y en los artículos de consenso para la estandarización de la DLCO publicados por la ATS y la ERS 5 .

Las ecuaciones seleccionadas se muestran en la Tabla 1.

\section{Sujetos}

Setenta y un sujetos normales sanos, no fumadores, con edades entre 18-88 años, 36 mujeres (50\%), habitantes de la región Metropolitana (520 metros sobre el nivel del mar), participantes en distintos protocolos de investigación de nuestro departamento, en los que se había realizado medición de DLCO como parte de su estudio, durante el período 2001-2009.

La medición de la DLCO se realizó de acuerdo a las normas del consenso ATS/ERS, con un sistema computarizado Sensormedics ${ }^{\mathrm{MR}}$ Vmax 2200 y 2800 , ambos con tecnología de lectura instantánea de monóxido de carbono. La mezcla de gas inspirado estaba constituida por monóxido de carbono $(0,3 \%)$, metano $(0,3 \%)$, oxígeno $(21 \%)$ y balance con nitrógeno. Los resultados fueron corregidos de acuerdo a la concentración de hemoglobina.

Para cada ecuación se calcularon los residuos según las normas ATS/ERS (medido-referencia), y se obtuvo la suma de estos.

\section{Análisis estadístico}

Para evaluar la normalidad de las variables continuas se realizó la prueba de KolmogorovSmirnov.

Para comparar el resultado de los sujetos con el valor de referencia para cada una de las ecuaciones se utilizó el programa GraphPad Prism 5 obteniendo correlación de Pearson y $\mathrm{r}^{2}$. Se consideró significativo un valor de $\mathrm{p}<0,05$.

\section{Resultados}

Las características que inciden en la variabilidad de cada ecuación se resumen en la Tabla 2. Se incluyen tanto características demográficas como características de la metodología utilizada.

Las variables continuas analizadas tienen distribución normal.

\section{a) Características demográficas}

Con respecto al género, en las ecuaciones seleccionadas, tanto hombres como mujeres están bien representados. En relación a la edad, sólo seis ecuaciones tienen grupos etarios amplios, entre 7 y 94 años, mientras que el resto aborda grupos específicos.

La mayoría de las ecuaciones fueron desarrolladas a partir de una muestra de sujetos nor- 
Tabla 1. Ecuaciones de referencia DLCO

\begin{tabular}{|c|c|}
\hline \multicolumn{2}{|l|}{ DLCO HOMBRES } \\
\hline 1961 Burrows et al & $36,7-0,238 * \mathrm{EDAD}$ \\
\hline 1982 Crapo RO et al ${ }^{9}$ & $0,416 *$ TALLA- $0,219 *$ EDAD-26,34 \\
\hline 1983 Miller $e t a l^{2}$ & $12,9113+0,418 *($ TALLA/2,54)-0,229*EDAD \\
\hline 1985 Paoletti et al ${ }^{10}$ & $-31,3822-0,1936 *$ EDAD $+0,441 *$ TALLA \\
\hline 1987 Knudson et al ${ }^{11}$ & $-11,3527-0,2741 *$ EDAD $+0,3551 *$ TALLA \\
\hline 1990 Roca et al ${ }^{12}$ & $0,3674 *$ TALLA-0,1961*EDAD-21,8982 \\
\hline 1993 (ERS) Cotes JE et al ${ }^{13}$ & $2,988536 *\left((11,11 *\right.$ TALLA $\left.\left./ 100)-\left(0,066^{*} \mathrm{EDAD}\right)-6,03\right)\right)$ \\
\hline 2008 B. Aguilaniu et $a l^{15}$ & Mayor 59 años: $-12,954+5,044+29,291 *($ TALLA/100)+EDAD*-0,262 \\
\hline & Menor 59 años: $-12,954+5,044+29,291 *($ TALLA/100)+EDAD*-0,161 \\
\hline 2008 B. Thompson et al ${ }^{14}$ & $1,109 *(\mathrm{TALLA} / 100)^{\wedge} 3-0,000402 * \mathrm{EDAD}^{\wedge} 2+4,696$ \\
\hline 2012 Francisco García-Río et al ${ }^{16}$ & $2,99 *\left(3,57-0,00000863 * \mathrm{EDAD}^{\wedge} 3+4,725 *(\mathrm{RAIZ}((\mathrm{TALLA} * \mathrm{PESO}) / 3600))\right)$ \\
\hline \multicolumn{2}{|l|}{ DLCO MUJERES } \\
\hline 1961 Burrows et $a l^{1}$ & $25-0,117 *$ EDAD \\
\hline 1982 Crapo RO et al ${ }^{9}$ & $0,256 *$ TALLA- $0,1444 *$ EDAD- 8,36 \\
\hline 1983 Miller et $a l^{2}$ & $2,2382+0,4068 *(\mathrm{TALLA} / 2,54)-0,1111 * \mathrm{EDAD}$ \\
\hline 1985 Paoletti et al ${ }^{10}$ & $5,0767-0,0677 *$ EDAD $+0,1569 *$ TALLA \\
\hline 1987 Knudson et $a l^{11}$ & $3,8821-0,146 *$ EDAD $+0,1872 *$ TALLA \\
\hline 1990 Roca et ll $^{12}$ & $0,1369 *$ TALLA- $0,1233 *$ EDAD $+0,0917 *$ PESO $+1,8879$ \\
\hline 1993 (ERS) Cotes JE et al ${ }^{13}$ & $2,988536 *((8,18 *$ TALLA/100)-(0,049*EDAD)-2,74) \\
\hline \multirow[t]{2}{*}{2008 B. Aguilaniu et al $^{15}$} & Mayor 59 años: $-12,954+29,291 *($ TALLA/100)+EDAD*-0,262 \\
\hline & Menor 59 años: $-12,954+29,291 *($ TALLA/100)+EDAD*-0,161 \\
\hline 2008 B. Thompson et al ${ }^{14}$ & $\begin{array}{l}(51,9 *(\text { TALLA/100)-3,901*(TALLA/100)^3+0,375*EDAD }+0,012 * \text { PESO- } \\
0,273 * \text { EDAD }^{*}(\text { TALLA/100)-57,703)*2,99 }\end{array}$ \\
\hline 2012 Francisco García-Río et al ${ }^{16}$ & $2,99 *\left(8,635-0,0736 * \mathrm{EDAD}+0,000001033 * \mathrm{TALLA}^{\wedge} 3\right.$ \\
\hline
\end{tabular}

Talla en cm, peso en $\mathrm{kg}$, edad en años.

Tabla 2. Comparación de ecuaciones de valores de referencia para DLCO

\begin{tabular}{|c|c|c|c|c|c|c|c|c|c|c|}
\hline & $\begin{array}{c}\text { Burrows }^{1} \\
1961\end{array}$ & $\begin{array}{c}\text { Crapo }^{9} \\
1981\end{array}$ & $\begin{array}{c}\text { Miller }^{2} \\
1983\end{array}$ & $\begin{array}{c}\text { Paoletti }^{10} \\
1985\end{array}$ & $\begin{array}{c}\text { Knudson }^{11} \\
1987\end{array}$ & $\begin{array}{c}\text { Roca }^{12} \\
1990\end{array}$ & $\begin{array}{c}\text { Cotes }^{13} \\
1993\end{array}$ & $\begin{array}{c}\text { Thomp- } \\
\text { son }^{14} \\
2008\end{array}$ & $\begin{array}{l}\text { Aguilaniu } \\
2008^{15}\end{array}$ & $\begin{array}{c}\text { García-Río } \\
2012^{16}\end{array}$ \\
\hline Hombres (n) & 84 & 123 & 249 & 243 & 99 & 233 & -- & 498 & 161 & 169 \\
\hline Mujeres (n) & 51 & 122 & 262 & 469 & 129 & 194 & -- & 474 & 142 & 262 \\
\hline Edad (años) & $<92$ & $15-91$ & $>18$ & $8-64$ & $7-85$ & $15-70$ & $18-70$ & $45-71$ & $18-94$ & $65-85$ \\
\hline Fumador & -- & no & no + ex + sí & no & no & notex & no+sí & notex & no & no \\
\hline $\begin{array}{l}\text { Respiración } \\
\text { única }\end{array}$ & -- & -- & -- & SI & SI & SI & SI & SI & SI & SI \\
\hline $\begin{array}{l}\text { Lectura } \\
\text { instantánea }\end{array}$ & NO & NO & NO & NO & NO & NO & NO & SI & NO & NO \\
\hline $\mathrm{FiO}_{2}$ & -- & 0,25 & 0,21 & 0,20 & 0,21 & 0,18 & $0,17-0,18$ & 0,206 & 0,21 & -- \\
\hline $\begin{array}{l}\text { Altitud } \\
\text { (metros) }\end{array}$ & -- & 1.400 & 176 & $\begin{array}{l}\text { Nivel del } \\
\text { mar }\end{array}$ & 700 & $\begin{array}{l}\text { Nivel del } \\
\text { mar }\end{array}$ & -- & $\begin{array}{l}\text { Nivel del } \\
\text { mar }\end{array}$ & $\begin{array}{c}300 y \\
30\end{array}$ & 655 \\
\hline Gas trazador & -- & Helio & Helio & Helio & Helio & Helio & Helio & Neón & Helio & Helio \\
\hline LIN & SI & SI & SI & SI & SI & SI & SI & SI & SI & SI \\
\hline $\begin{array}{l}\text { Suma } \\
\text { residuos }\end{array}$ & 271 & -239 & 25 & -272 & -350 & $-53,4$ & 22 & 81,6 & $-91,8$ & -49 \\
\hline Pearson $r$ & 0,797 & 0,844 & 0,844 & 0,824 & 0,845 & 0,842 & 0,843 & 0,825 & 0,825 & 0,815 \\
\hline IC 95\% & $0,69-0,86$ & $0,76-0,89$ & $0,76-0,89$ & $0,73-0,88$ & $0,76-0,89$ & $0,76-0,89$ & $0,76-0,89$ & $0,73-0,88$ & $0,73-0,88$ & $0,72-0,87$ \\
\hline Valor $\mathrm{p}$ & $<0,0001$ & $<0,0001$ & $<0,0001$ & $<0,0001$ & $<0,0001$ & $<0,0001$ & $<0,0001$ & $<0,0001$ & $<0,0001$ & $<0,0001$ \\
\hline $\mathrm{r}^{2}$ & 0,6362 & 0,7130 & 0,7134 & 0,6792 & 0,7141 & 0,7099 & 0,7115 & 0,6808 & 0,6815 & 0,6656 \\
\hline
\end{tabular}

LIN: límite inferior de normalidad. 
males y sanos; sin embargo, en dos de ellas $\left(\right.$ Miller $^{2}$ y Paoletti ${ }^{10}$ ) la población estudiada incluía sujetos expuestos a contaminantes ambientales.

Cuatro de las ecuaciones fueron obtenidas a partir de una muestra de sujetos que incluía ex fumadores, mientras que dos ecuaciones incluían sujetos que continuaban fumando.

La ecuación de la ERS (Cotes 1993) ${ }^{13}$, corresponde a una recopilación de datos de varios grupos europeos, que cumplían con criterios de calidad.

La altitud informada por los distintos trabajos fue también variable, con algunos trabajos realizados a nivel del mar $\left(\right.$ Paoletti $^{10}$, Roca ${ }^{12}$ y Thompson ${ }^{14}$ ) y uno a 1.400 metros sobre nivel del mar (Crapo $)^{9}$.

\section{b) Características de la metodología utilizada}

Desde 1985 se ha utilizado el método de la respiración única, en las publicaciones anteriores no se describe el método utilizado pudiendo ser el de múltiples respiraciones.

Nueve de las 10 ecuaciones que se muestran en la Tabla 2, no utilizan la metodología más moderna y de menor variabilidad, para la medición de la concentración de gases en la muestra alveolar. Sólo la ecuación de Thompson y cols. ${ }^{14}$ fue obtenida con equipamiento que permite la lectura instantánea de monóxido de carbono, pero su muestra se encuentra acotada a individuos con edades entre los 45 y los 71 años y no excluye ex fumadores.

Con respecto a la concentración de oxígeno en la mezcla inspirada, las ecuaciones difieren en la fracción inspirada, muchas veces con el objetivo de corregir una presión inspirada de oxígeno disminuida por efecto de la altitud a la que se realizó el estudio. Es así como la fracción inspirada de oxígeno varía entre 0,17 y 0,25 .

Por último, también existen diferencias en el gas trazador utilizado, ocho ecuaciones usaron helio como gas trazador.

\section{c) Residuos y correlación}

La Figura 1 muestra el promedio \pm error estándar de la suma de los residuos para cada ecuación.
La correlación de Pearson y $\mathrm{r}^{2}$ para cada ecuación se muestran en la Tabla 2.

Las ecuaciones que presentan la menor suma de residuos son las de Miller ${ }^{2}$, Roca ${ }^{12}$ y Cotes $^{13}$.

\section{Comentarios}

Esta revisión incorpora el mayor número de ecuaciones de referencia de DLCO disponibles en la literatura. Los resultados del análisis de estas ecuaciones muestran que éstas tienen limitaciones significativas al momento de elegir una de ellas para la interpretación de los resultados de DLCO de los pacientes que acuden a los laboratorios de función pulmonar.

Si bien las recomendaciones internacionales indican elegir la ecuación con la menor suma de residuos ${ }^{4}$, por ser en cierta forma "más cercana" a los resultados locales, no parece razonable escoger una ecuación de acuerdo a esta recomendación, si ésta fue obtenida de manera muy diferente a la forma en que se obtienen los valores de DLCO a interpretar.

Observamos que la mayoría de las ecuaciones disponibles utilizó una metodología de alta variabilidad, es decir no fueron obtenidas a partir de mediciones en equipos con lectura instantánea de $\mathrm{CO}$ y gas trazador. La única excepción la constituye la ecuación de Thompson et $\mathrm{al}^{14}$, que incorpora lectura instantánea de monóxido de carbono, con baja variabilidad en las mediciones. Sin embargo, esta

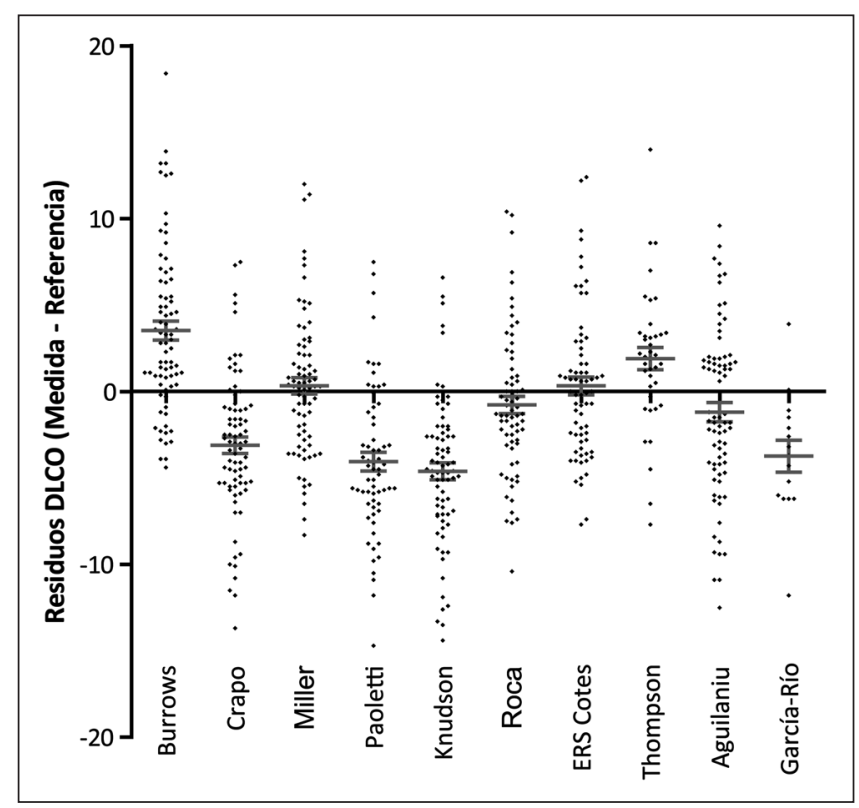

Figura 1. Residuos (valor observado- valor referencia) para las distintas ecuaciones de referencia de DLCO (puntos). Promedio \pm error estándar (líneas horizontales). En el eje horizontal se señala el primer autor de cada una de las 10 ecuaciones de referencia comparadas en las Tablas 1 y 2 . 
ecuación presenta una suma de residuos elevada al compararla con la población de este estudio.

Las tres ecuaciones que se podrían seleccionar según los criterios ATS/ERS por tener las menores sumas de residuos, son la de Miller ${ }^{2}$, Roca ${ }^{12}$ y Cotes ${ }^{13}$; pero éstas incluyen sujetos fumadores y ex fumadores, y además difieren en la fracción inspirada de oxigeno utilizada.

Para la realidad nacional, es necesario desarrollar una ecuación en individuos normales y sanos no fumadores de grupos etarios representativos y con una metodología que asegure una baja variabilidad.

\section{Agradecimientos}

Los autores agradecen a todos los funcionarios del laboratorio de función pulmonar de la Pontificia Universidad Católica de Chile que hicieron posible la realización del presente trabajo de investigación.

\section{Bibliografía}

1.- BURROWS B, KASIK J E, NIDEN A H, BARCLAY W R. Clinical usefulness of the single-breath pulmonary diffusing capacity test. Am Rev Respir Dis 1961; 84: 789-806.

2.- MILLER A, THORNTON J C, WARSHAW R, ANDERSON H, TEIRSTEIN A S, SELIKOFF I J. Singlebreath diffusing capacity in a representative sample of the population of Michigan, a large industrial state. Predicted values, lower limits of normal, and frequencies of abnormality by smoking history. Am Rev Respir Dis 1983; 127: 270-7.

3.- MOHSENIFAR Z, LEE S M, DÍAZ P, CRINER G, SCIURBA F, GINSBURG M, WISE R A. Single-breath diffusing capacity of the lung for carbon monoxide: a predictor of $\mathrm{PaO}_{2}$, maximum work rate, and walking distance in patients with emphysema. Chest 2003; 123: 1394-400.

4.- American Thoracic Society. Single-breath carbon monoxide diffusing capacity (transfer factor). Recommendations for a standard technique: 1995 update. Am J Respir Crit Care Med 1995; 152 (6 Pt1): 2185-98.

5.- MACINTYRE N, CRAPO RO, VIEGI G, JOHNSON DC, VAN DER GRINTEN CP, BRUSASCO V, et al. Standardization of the single-breath determination of carbon monoxide uptake in the lung. Eur Respir J 2005; 26: 720-35.

6.- Single breath carbon monoxide diffusing capacity (transfer factor). Recommendations for a standard technique. Statement of the American Thoracic Society. Am Rev Respir Dis 1987; 136: 1299-307.
7.- WANGER J, IRVIN C. Comparability of pulmonary function results from 13 laboratories in a metropolitan area. Respir Care 1991; 36: 1375-82.

8.- PELLEGRINO R, VIEGI G, BRUSASCO V, CRAPO R O, BURGOS F, CASABURI R, et al. Interpretive strategies for lung function tests. Eur Respir J 2005; 26 : 948-68.

9.- CRAPO R O, MORRIS A H. Standardized single breath normal values for carbon monoxide diffusing capacity. Am Rev Respir Dis 1981; 123: 185-9.

10.- PAOLETTI P, VIEGI G, PISTELLI G, DI PEDE F, FAZZI P, POLATO R, et al. Reference equations for the single-breath diffusing capacity. A cross-sectional analysis and effect of body size and age. Am Rev Respir Dis 1985; 132: 806-13.

11.- KNUDSON R J, KALTENBORN W T, KNUDSON D E, BURROWS B. The single-breath carbon monoxide diffusing capacity. Reference equations derived from a healthy non-smoking population and effects of hematocrit. Am Rev Respir Dis 1987; 135: 805-11.

12.- ROCA J, RODRÍGUEZ-ROISIN R, COBO E, BURGOS F, PÉREZ J, CLAUSEN J L. Single-breath carbon monoxide diffusing capacity prediction equations from a Mediterranean population. Am Rev Respir Dis 1990; 14 (4 Pt 1): 1026-32.

13.- COTES J E, CHINN D J, QUANJER P H, ROCA J, YERNAULT J C. Standardization of the measurement of transfer factor (diffusing capacity). Report Working Party Standardization of Lung Function Tests, European Community for Steel and Coal. Official Statement of the European Respiratory Society. Eur Respir J Suppl 1993; 16: 41-52.

14.- THOMPSON B R, JOHNS D P, BAILEY M, RAVEN J, WALTERS E H, ABRAMSON M J. Prediction equations for single breath diffusing capacity (Tlco) in a middle aged caucasian population. Thorax 2008; 63: 889-93.

15.- AGUILANIU B, MAITRE J, GLÉNET S, GEGOUTPETIT A, GUÉNARD H. European reference equations for CO and NO lung transfer. Eur Respir J 2008; 31: 1091-7.

16.- GARCÍA-RÍO F, DORGHAM A, GALERA R, CASITAS R, MARTÍNEZ E, ALVAREZ-SALA R, PINO J M. Prediction equations for single-breath diffusing capacity in subjects aged 65 to 85 years. Chest 2012; 142: 175-84.

\section{Correspondencia a:}

Dra. Catalina Paz Briceño Villafañe

Departamento de Enfermedades Respiratorias

Pontificia Universidad Católica de Chile

Marcoleta 350 piso 1 interior, Santiago, Chile

Email: catalinabriceno@gmail.com 